\title{
Rapidity profiles from 3+1D Glasma simulations with finite longitudinal thickness
}

\author{
Andreas Ipp and David Müller* \\ Institute for Theoretical Physics, TU Wien, \\ Wiedner Hauptstraße 8-10, A-1040 Vienna, Austria \\ E-mail: ipp@hep.itp.tuwien.ac.at, david.mueller@tuwien.ac.at
}

\begin{abstract}
We present our progress on simulating the Glasma in the early stages of heavy ion collisions in a non-boost-invariant setting. Our approach allows us to describe colliding nuclei with finite longitudinal width by extending the McLerran-Venugopalan model to include a parameter for the Lorentz-contracted but finite extent of the nucleus in the beam direction. We determine the rapidity profile of the Glasma energy density, which shows strong deviations from the boost invariant result. Both broad and narrow profiles can be produced by varying the initial conditions. We find reasonable agreement when we compare the results to rapidity profiles of measured pion multiplicities from RHIC.
\end{abstract}

The European Physical Society Conference on High Energy Physics

5-12 July

Venice, Italy

\footnotetext{
* Speaker.
} 


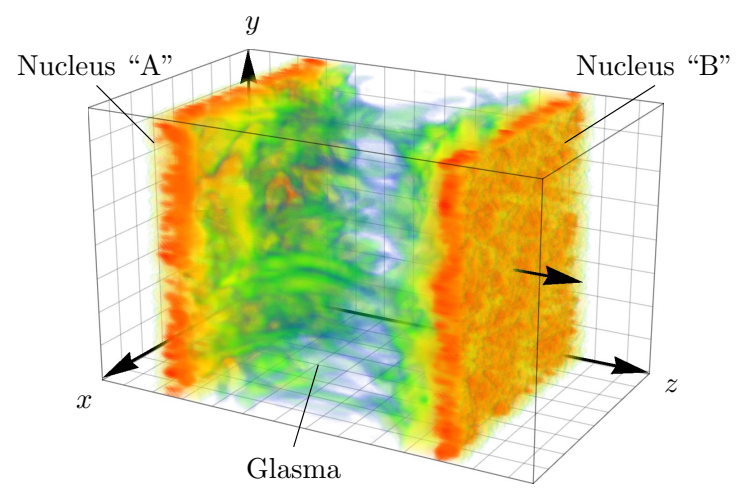

Figure 1: A 3D plot of the energy density directly after the collision, showing both nuclei " $A$ " and "B" and the Glasma with visible flux tube structure [1]. The box only covers a small part of the full collision in the transverse plane spanned by $x$ and $y$, for which we use periodic boundary conditions as opposed to fixed boundary conditions in the longitudinal direction.

\section{Introduction}

In the earliest stages of heavy ion collisions the color glass condensate (CGC) effective field theory $[2,3]$ provides a first principles, weak coupling, yet non-perturbative description in terms of classical Yang-Mills fields and color currents. The main idea is a separation of degrees of freedom in a fast nucleus into partons with large longitudinal momentum fraction $x$ (e.g. valence quarks) and partons with small $x$ (mostly soft gluons). Large $x$ partons are assumed to be randomly distributed, recoilless classical color charges, moving at the speed of light, while small $x$ partons are described as classical gluon fields sourced by the large $x$ partons. In the ultrarelativistic limit the classical field of the soft gluons becomes a shock wave. The result of a collision of two such shock waves is a field initially composed of purely longitudinal color flux tubes known as the Glasma [4], a precursor to the quark gluon plasma. Due to the assumption of infinitely thin shock waves the Glasma is boost invariant, i.e. there is no dependence on space-time rapidity $\eta_{s}$. After the collision the Glasma evolves and expands classically according to the Yang-Mills equations, preserving the initial boost invariance. This classical evolution only holds for a short amount of time, up to roughly $\tau \lesssim 1 \mathrm{fm} / c$, after which the field quickly becomes decoherent and the classical approach is considered to be invalid.

The evolution of the boost invariant Glasma and its properties have been investigated thoroughly both numerically $[5,6,7,8]$ and analytically in the weak field limit [9] or using the small $\tau$ expansion [10]. The Glasma can also be studied in settings without boost invariance, for instance by introducing small, rapidity dependent fluctuations in the initial conditions [11, 12], by considering the saturation scale as a function of rapidity [13] or more fundamentally at next-to-leading order in the gauge coupling, by making use of the JIMWLK evolution [14, 15, 16]. However, in all of these approaches one still uses the Glasma initial conditions [17], which are derived under the assumption of boost invariance and are thus not strictly valid at finite collision energy.

Obviously, realistic nuclei at finite collision energies are not infinitely thin. The finite "classical" longitudinal extent of a nucleus at a given velocity $v$ is proportional to $R / \gamma$, where $R$ is the 
radius of the nucleus and $\gamma^{-1}=\sqrt{1-(v / c)^{2}}$ is the Lorentz factor. In the context of the JIMWLK evolution, the longitudinal extent of nuclei might even be larger than the classical extent: as the evolution to lower longitudinal momenta ( $\operatorname{smaller} x$ ) progresses, more and more small $x$ gluons are added to the gluon field of the nucleus. Due to uncertainty in the longitudinal position at small $x$, these gluons are spread out over a length typically larger than $R / \gamma$, which leads to a picture of rather thick nuclei even at ultrarelativistic energies [18]. Except for a few pioneering studies [19, 20], the effects of finite longitudinal extent and its consequences on the evolution of the Glasma have been largely ignored in the past.

The aim of this work is to extend the CGC/Glasma description to include the finite longitudinal extent of the colliding nuclei, striving for a more realistic picture at finite collision energies. In this proceedings contribution we present our progress in simulating heavy-ion collisions in the CGC framework at finite collision energies based on our previous publications [21, 1].

\section{Initial conditions}

In the standard picture of a boost invariant collision the color current of a left moving nucleus (here denoted as "A") can be written as

$$
J_{(A)}^{-}\left(x^{+}, x_{T}\right)=\delta\left(x^{+}\right) \rho_{(A)}\left(x_{T}\right),
$$

where $x^{ \pm} \equiv\left(x^{0} \pm x^{3}\right) / \sqrt{2}$ and $\rho_{(A)}\left(x_{T}\right)$ is the color charge density in the transverse plane. Supplied with the covariant gauge condition $\partial_{\mu} A^{\mu}(x)=0$, the current generates a field given by

$$
A_{(A)}^{-}\left(x^{+}, x_{T}\right)=-\Delta_{T}^{-1} J_{(A)}^{-}\left(x^{+}, x_{T}\right)
$$

with the Laplace operator in the transverse plane $\Delta_{T}$. Likewise, a right moving nucleus ("B") is described by the color current $J_{(B)}^{+}\left(x^{-}, x_{T}\right)$ and the field $A_{(B)}^{+}\left(x^{-}, x_{T}\right)$. As mentioned in the introduction, collisions of two such fields leads to the boost invariant Glasma picture. In order to describe the non-boost-invariant scenario where nuclei have finite longitudinal extent we have to relax the assumption made in Eq. (2.1) and account for extended support of the color charges along the longitudinal direction. The solution to the field equations before the collision in Eq. (2.2) remain unchanged. In particular we look at initial conditions where the longitudinal shape can be separated from the transverse color charge density

$$
J_{(A)}^{-}\left(x^{+}, x_{T}\right)=f\left(x^{+}\right) \rho_{(A)}\left(x_{T}\right),
$$

where $f\left(x^{+}\right)$is a normalized function that defines the longitudinal profile. The form of the current is a special case where non-trivial color structure in the longitudinal extent is neglected [22]. With extended currents and fields it is no longer possible to just focus on the forward light cone. Instead, we solve the full Yang-Mills equations

$$
D_{\mu} F^{\mu v}(x)=J_{(A)}^{v}(x)+J_{(B)}^{v}(x)
$$

in the laboratory frame in $3+1$ dimensions. Working the lab frame forces us to explicitly include the color currents in our simulation and we have to make sure that the non-Abelian charge conservation 


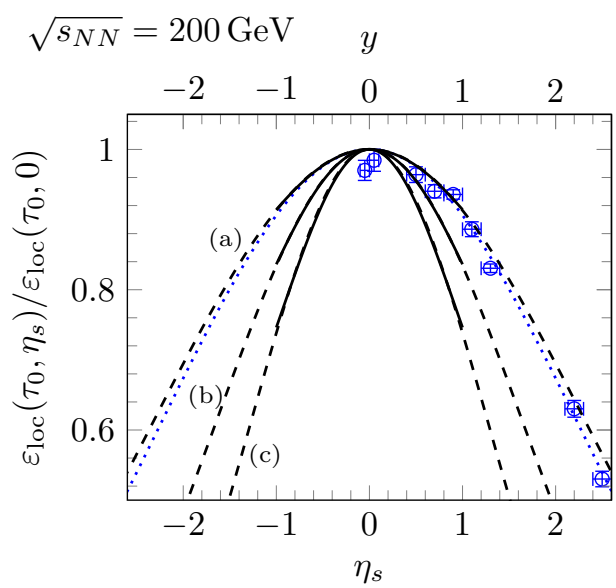

$$
\sqrt{s_{N N}}=200 \mathrm{GeV}, \quad m=200 \mathrm{MeV}
$$

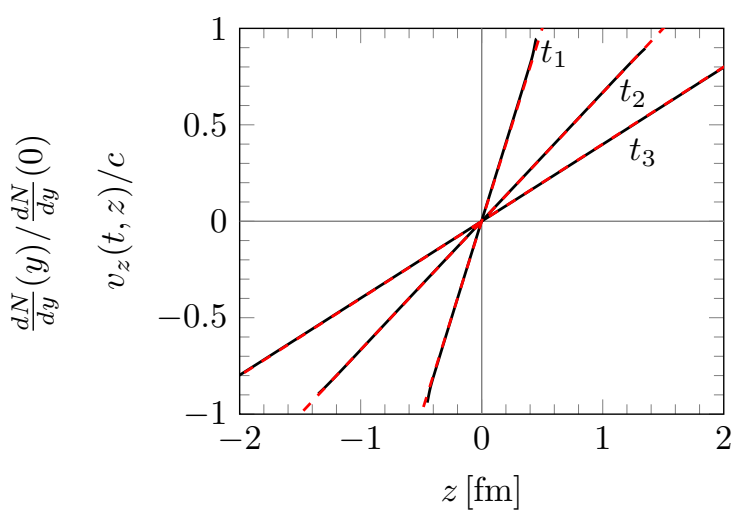

Figure 2: Left: Comparison of the space-time rapidity profile of the LRF energy density $\varepsilon_{l o c}\left(\tau_{0}, \eta_{s}\right)$ (thick solid lines), $\pi^{+}$multiplicity $d N / d y$ at RHIC [24] (data points) and Gaussian fits (dashed and dotted). The infrared regulator $m$ has a large effect on the width: (a) $m=0.2 \mathrm{GeV}$, (b) $m=0.4 \mathrm{GeV}$ and (c) $m=0.8 \mathrm{GeV}$. Right: Longitudinal velocity $v_{z}(t, z)$ as a function of the longitudinal coordinate $z$ evaluated at different laboratory frame times $t$ from out simulation (black, solid lines) compared to the free-streaming case $v_{z}(t, z)=z / t$ (red, dashed lines). We show three different times: $t_{1}=0.5 \mathrm{fm} / c, t_{2}=1.5 \mathrm{fm} / c$ and $t_{3}=2.5 \mathrm{fm} / c$.

$D_{\mu} J_{(A, B)}^{\mu}(x)=0$ holds. Details of how we do this numerically are described in [21]. As a model for large nuclei we use the McLerran-Venugopalan model [23] defined by the charge density correlator

$$
\left\langle\rho_{(A, B)}^{a}\left(x_{T}\right) \rho_{(A, B)}^{b}\left(y_{T}\right)\right\rangle=g^{2} \mu^{2} \delta^{(2)}\left(x_{T}-y_{T}\right) \delta^{a b},
$$

where $g$ is the gauge coupling constant and $\mu$ controls the average charge fluctuations in the transverse plane. In our approach (Eq. (2.3)) the two-dimensional charge density given by Eq. (2.5) is simply extended in the longitudinal direction. We relate the width of $f\left(x^{+}\right)$(which we take to be a Gaussian) to the Lorentz contracted diameter of the nucleus $2 R / \gamma$, which in turn is related to the collision energy $\sqrt{s_{N N}}$. In our most recent publication [1] we look at RHIC-like scenarios of central $\mathrm{Au}+\mathrm{Au}$ collisions with $\sqrt{s_{N N}}$ at $200 \mathrm{GeV}$ and $130 \mathrm{GeV}$.

\section{Results}

Solving the field equations for the collision scenario in $3+1$ we obtain pictures like Fig. 1, where we plot the energy density of the Yang-Mills fields directly after the collision. We study the Glasma in our simulations by looking at the local rest frame (LRF) energy density $\varepsilon_{l o c}\left(\tau, \eta_{s}\right)$ which we compute by diagonalizing the energy-momentum tensor $T_{v}^{\mu}(x)$. In Fig. 2 (left) we show $\varepsilon_{\text {loc }}\left(\tau_{0}, \eta_{s}\right)$ as a function of space-time rapidity at $\tau_{0}=1 \mathrm{fm} / c$ for $\sqrt{s_{N N}}=200 \mathrm{GeV}$. We observe that these rapidity profiles are approximately Gaussian in shape. The width of the profiles depends on the energy $\sqrt{s_{N N}}$ and also strongly on the infrared regulator that is used in the initial conditions. As one should expect, we observe that reducing $\sqrt{s_{N N}}$, thus increasing the longitudinal extent, the profiles become more narrow. The effect of the infrared regulator is more surprising, not well understood and warrants further in-depth studies. The width itself is rather independent of evaluation time $\tau_{0}$ as long as $\tau_{0} \gtrsim 0.3 \mathrm{fm} / c$ after which one enters the free-streaming limit. 
Free-streaming can be observed by looking at the longitudinal velocity $v_{z}$, which corresponds to the velocity associated with the Lorentz boost that transforms from the laboratory frame into the LRF. In Fig. 2 (right) we plot $v_{z}(t, z)$ as a function of the longitudinal coordinate $z$ at different times $t$ and compare it to the free-streaming case, where $v_{z}=z / t$. We observe that the two curves match, which implies that the LRF mostly corresponds to the $\left(\tau, \eta_{s}\right)$ frame. The free-streaming limit is also visible in the strong pressure anisotropy at later times where transverse pressure $p_{T}$ dominates longitudinal pressure $p_{L}$.

The conclusion we can draw from these observations is that by including finite longitudinal extent, thus explicitly breaking the boost invariance of the system, we obtain non-flat rapidity profiles that develop early on in the evolution, but the Glasma still flows in a free-streaming manner just like in the boost invariant approximation. Finite longitudinal extent therefore does not fundamentally change the picture of the boost invariant Glasma, except for non-flat rapidity profiles.

\section{Acknowledgments}

The authors thank A. Kurkela and T. Lappi for helpful discussions. This work has been supported by the Austrian Science Fund FWF, Project No. P26582-N27 and Doctoral program No. W1252-N27. The computational results have been achieved using the Vienna Scientific Cluster.

\section{References}

[1] A. Ipp and D. Müller, Broken boost invariance in the Glasma via finite nuclei thickness, Phys. Lett. B771 (2017) 74-79, [1703.00017].

[2] F. Gelis, Color Glass Condensate and Glasma, Int. J. Mod. Phys. A28 (2013) 1330001, [1211.3327].

[3] F. Gelis, E. Iancu, J. Jalilian-Marian and R. Venugopalan, The Color Glass Condensate, Ann. Rev. Nucl. Part. Sci. 60 (2010) 463-489, [1002.0333].

[4] T. Lappi and L. McLerran, Some features of the glasma, Nucl. Phys. A772 (2006) 200-212, [hep-ph/0602189].

[5] A. Krasnitz and R. Venugopalan, Nonperturbative computation of gluon minijet production in nuclear collisions at very high-energies, Nucl. Phys. B557 (1999) 237, [hep-ph/ 9809433$].$

[6] T. Lappi, Production of gluons in the classical field model for heavy ion collisions, Phys. Rev. C67 (2003) 054903, [hep-ph/0303076].

[7] T. Lappi, Energy density of the glasma, Phys. Lett. B643 (2006) 11-16, [hep-ph / 0606207$].$

[8] A. Dumitru, Y. Nara and E. Petreska, Magnetic flux loop in high-energy heavy-ion collisions, Phys. Rev. D88 (2013) 054016, [1302.2064].

[9] H. Fujii and K. Itakura, Expanding color flux tubes and instabilities, Nucl. Phys. A809 (2008) $88-109,[0803.0410]$.

[10] G. Chen, R. J. Fries, J. I. Kapusta and Y. Li, Early Time Dynamics of Gluon Fields in High Energy Nuclear Collisions, Phys. Rev. C92 (2015) 064912, [1507.03524]. 
[11] P. Romatschke and R. Venugopalan, The Unstable Glasma, Phys. Rev. D74 (2006) 045011, [hep-ph/0605045].

[12] K. Fukushima and F. Gelis, The evolving Glasma, Nucl. Phys. A874 (2012) 108-129, [1106. 1396 ].

[13] T. Lappi, Rapidity distribution of gluons in the classical field model for heavy ion collisions, Phys. Rev. C70 (2004) 054905, [hep-ph/ 0409328$].$

[14] E. Iancu, A. Leonidov and L. D. McLerran, Nonlinear gluon evolution in the color glass condensate. 1., Nucl. Phys. A692 (2001) 583-645, [hep-ph/ 0011241$].$

[15] H. Weigert, Evolution at small $x_{b j}$ : The Color Glass Condensate, Prog. Part. Nucl. Phys. 55 (2005) 461-565, [hep-ph/0501087].

[16] B. Schenke and S. Schlichting, 3D glasma initial state for relativistic heavy ion collisions, Phys. Rev. C94 (2016) 044907, [1605.07158].

[17] A. Kovner, L. D. McLerran and H. Weigert, Gluon Production from Non-Abelian Weizsäcker-Williams Fields in Nucleus-Nucleus Collisions, Phys.Rev. D52 (1995) 6231-6237, [hep-ph / 9502289 ].

[18] E. Iancu and D. N. Triantafyllopoulos, JIMWLK evolution in the Gaussian approximation, JHEP 04 (2012) 025, [1112.1104].

[19] W. Poschl and B. Muller, Real time dynamics of colliding gauge fields and the 'glue burst', Phys. Rev. D60 (1999) 114505, [nucl-th/9812066].

[20] Ş. Özönder and R. J. Fries, Rapidity Profile of the Initial Energy Density in Heavy-Ion Collisions, Phys. Rev. C89 (2014) 034902, [1311.3390].

[21] D. Gelfand, A. Ipp and D. Müller, Simulating collisions of thick nuclei in the color glass condensate framework, Phys. Rev. D94 (2016) 014020, [1605.07184].

[22] K. Fukushima, Randomness in infinitesimal extent in the McLerran-Venugopalan model, Phys. Rev. D77 (2008) 074005, [0711.2364].

[23] L. D. McLerran and R. Venugopalan, Gluon distribution functions for very large nuclei at small transverse momentum, Phys. Rev. D49 (1994) 3352-3355, [hep-ph/9311205].

[24] BRAHMS collaboration, I. G. Bearden et al., Charged meson rapidity distributions in central $A u+A u$ collisions at $\sqrt{s_{N N}}=200 \mathrm{GeV}$, Phys. Rev. Lett. 94 (2005) 162301, [nucl-ex/ 0403050 ]. 